\title{
Confrontations et convergences éthiques entre marketing et information autour de la publicité native
}

\author{
Baillargeon, Dany, Ph.D. \\ Professeur, Université de Sherbrooke (Canada) \\ dany.baillargeon@usherbrooke. \\ Coutant, Alexandre, Ph.D. \\ Professeur, Université du Québec à Montréal \\ (Canada) \\ coutant.alexandre@uqam.ca \\ Carignan, Marie-Eve, Ph.D. \\ Professeure, Université de Sherbrooke (Canada) \\ marie-eve.carignan@usherbrooke.ca \\ Dionne, Elyse \\ Étudiante à la maîtrise en communication, \\ Université de Sherbrooke (Canada) \\ elyse.dionne@usherbrooke.ca \\ Tourigny, Mikaëlle \\ Étudiante à la maîtrise en communication, \\ Université de Sherbrooke (Canada) \\ mikaelle.tourigny@usherbrooke.ca
}




\title{
Résumé
}

La perméabilité de la division information/publicité dans les salles de rédaction fait récursivement l'objet de débats au sein de l'industrie journalistique. Mus par des logiques différentes, univers marketing et journalistique sont supposés se prémunir de toute influence mutuelle. L'arrivée des plateformes socio numériques, l'éclatement du modèle économique des médias d'information et l'irritation face à l'intrusion publicitaire et les stratégies de blocage encouragent de nouveaux chevauchements, dont la publicité native (PN). Les différentes justifications ou critiques de cette technique controversée constituent une entrée pour analyser le rapport à l'éthique des deux professions, journalisme et marketing. Cette communication propose d'interroger ce phénomène par une double analyse. D'abord en repérant dans le discours des professionnels du marketing et de l'information les différentes rationalités entourant l'utilisation, ou non, de la PN. Ces rationalités sont ensuite adossées aux codes, chartes et guides déontiques. Au final, nous arguons que les guides actuels sont insuffisants pour encadrer le bouleversement professionnel qu'engendre la PN, entre autres sous la force de tensions séculaires, mais fortement bousculées par des rationalités convergentes. Ce paradoxe semble se solder par l'arrivée d'un nouveau type de professionnel, le producteur de contenu.

Mots Clés : publicité native, journalisme, analyse de discours, éthique

\begin{abstract}
The porousness of the information/advertising division in newsrooms is a recursive topic of debate in the journalism industry. With their distinct logics, the worlds of marketing and journalism are supposed to guard against any mutual influence, as enshrined in ethical codes and charters. But the rise of social media platforms, the fragmentation of the economic model of the news media, the irritation of advertising intrusion, and the ad blocking software are encouraging new overlaps, one being native advertising (NA). The various justifications or criticisms of this controversial technique constitute for us the entry point to analyze the ethics of both journalism and marketing in regard of NA. First, we identify, within the discourse of both marketing and information professionals, the different rationalities surrounding the use or non use of NA. These rationalities are then compared to codes, charters and deontic guides in use. In the end, we argue that the current guides are insufficient to frame the professional upheaval that NA generates, under the force of deep-rooted tensions, now strongly shaken by convergent economical rationalities. This paradox seems to result in the arrival of a new type of professional, the content producer.
\end{abstract}

Keywords: Ethics, deontology, native advertising, marketing, journalism 


\begin{abstract}
« Il s'avère également que les tentatives d'intrusion commerciale et publicitaire dans les contenus sont plus agressives que jamais. » (Saulnier, 2015)

«Demain, votre marque sera un média » (Henrard \& Pierra, 2015, p. 13)

«[...] dans une large part, la définition de la publicité native dépend du siège dans lequel est assise la personne qui tente de la décrire. » (IAB, 2013, p. 2)
\end{abstract}

La perméabilité de la division information/publicité dans les salles de rédaction fait récursivement l'objet de débats au sein de l'industrie journalistique. Mus par des logiques différentes, univers marketing et journalistique sont supposés se prémunir de toute influence mutuelle (Toussaint-Desmoulins, 2015). Ce principe est officiellement reconnu dans les chartes encadrant la pratique des deux professions en raison des enjeux démocratiques qu'il soulève (Floris, 2003). Néanmoins, des genres mitoyens ont toujours calqué les formes journalistiques et brouillé les schèmes de représentations des consommateurs (Moumouni, 2005; Seligman, 2015; Wright, 1986).

La remise en cause de la séparation entre information et promotion est d'autant facilitée que les publicitaires entretiennent un rapport variable aux règles éthiques encadrant leur pratique, relâchement fondé sur une faible formation à ses enjeux, une vision technique de leur métier et une rationalisation économique des effets de leurs actions (Cossette, 2009; Laczniak, 1993) justifiant une «myopie » et un « mutisme moraux » (Drumwright, Murphy, 2004).

Qui plus est, l'arrivée des plateformes socio numériques, l'éclatement du modèle économique des médias d'information et l'érosion du lectorat encouragent de nouveaux chevauchements. De fait, alors que les médias d'information dits traditionnels font face à une crise de leurs modèles d'affaires - revenus publicitaires et lectorat en chute libre, consommateurs réticents à payer pour du contenu d'information, arrivée des géants de la technologie numérique dans l'édition de contenu d'information (Newman, et al., 2015) -, les annonceurs, eux, voient leurs investissements publicitaires se buter à des consommateurs irrités par la communication marketing (Rosenwald, 2015; Shields, 2016; WARC, 2016a) et aux bloqueurs de publicité. Dans les deux camps, une perte de revenu s'ensuit, sans compter les contrecoups et remises en question de leur industrie (Drohan, 2016; WARC, 2015).

C'est dans cette mouvance qu'arrive la publicité native (PN). Le terme est apparu en 2012 pour désigner un type particulier de petites annonces uniques et indigènes à l'expérience de lecture dans un média. Plusieurs grandes marques de médias - telles que The Atlantic, The Guardian, The New York Time, Vice.com, The Wall Street Journal, Yahoo!, Wired, etc. - ont commencé à avoir recours plus massivement à la publicité native depuis 2014 (Dienel \& Robinson, 2015; Manic, 2015). Certes, cette forme d'émulation du contenu journalistique n'est pas nouvelle (Seligman, 2015) : depuis la fin du $19^{\mathrm{e}}$ siècle, « le monde des affaires [tend] à recourir à des formes de publicité sous le couvert de l'information » (Moumouni, 2005, p. 139) de façon à en 
légitimer la validité aux yeux des consommateurs. Des consumers magazines aux catalogues, publireportage et autres guides Michelin, la publicité déguisée a cours depuis longtemps (Eveno, 2004; Martin, 2004; Moumouni, 2005; Palmer, 2004). Toutefois, ne fut-ce que pour sa portée, la publicité native n'est pas un épiphénomène ni une stratégie marginale. De fait, en Europe, les dépenses dans ce domaine s'élevaient à environ 5 milliards d'euros en 2015. Pour 2020, les prévisions tablent sur plus de 13 milliards. La PN comptera alors pour près de $50 \%$ des investissements en numérique (WARC, 2016b). Aux États-Unis, les investissements en publicité native n'ont cessé d'augmenter depuis 2012, représentant le quart des investissements en communications numériques (Shields, 2015).

Cette forme de publicité est dite « native » puisqu'elle s'encastre dans le design et l'ergonomie aux supports qui l'accueillent, au lieu d'être reléguée aux espaces publicitaires traditionnels. Souvent proche de la ligne éditoriale du titre, ce «contenu de marque» suppose une approche journalistique de la promotion. L'intégration de la PN dans l'écosystème médiatique et marketing demeure floue : des salles de rédaction mettent leurs journalistes au service des marques, parfois dans des pôles en marge de la rédaction, parfois non, avec ou sans pigistes, alors que les marques deviennent elles-mêmes des productrices de contenu (Henrard \& Pierra, 2015; Lefebvre, 2014; Le Goff, et al., 2014). Par conséquent, les organismes régulant la pratique publicitaire et journalistique peinent à suivre la cadence, tentant de border «l'irresponsabilité, non seulement éventuelle, mais bien plutôt probable, des divers acteurs du secteur [du numérique] » (Devèze, 2005, p. 26).

Cette communication propose d'explorer les tensions éthiques générées par la PN. Dans un premier temps, nous proposons un recadrage de ce que l'on entend par publicité native, ses contours étant plutôt flous. Puis, à travers une analyse des discours professionnels tant marketing que journalistique, nous mettons en lumière les différentes rationalités qui entourent la PN. Dit autrement, de quelle façon justifie-t-on ou non l'utilisation de la PN dans chacun des camps? Par une catégorisation et un mappage de ces discours, nous proposons, dans un troisième temps, d'illustrer les zones de convergences et de divergences de ces rationalités, pour mieux les adosser aux codes, chartes et guides déontiques. Au final, nous arguons que ces derniers sont inaptes à rendre compte du bouleversement professionnel engendré par l'arrivée massive de la PN. Nous proposons alors quelques pistes de solutions et ouvrons sur différentes perspectives de recherche autour de ce « phénomène » venant brouiller les cartes professionnelles.

\section{Les contours agilement flous de la publicité native}

Dans son livre blanc consacré à la publicité native, l'International Advertising Bureau souligne ainsi qu' « il n'existe aucune définition universelle, puisque dans 
une large part, la définition de la publicité native dépend du siège dans lequel est assise la personne qui tente de la décrire. » (IAB, 2013, p. 2. Notre traduction).

Lorsque l'on tente de décrire ce qu'est la publicité native, deux vecteurs nous permettent de prendre en compte sa spécificité : l'intégration et le contenu. Du côté de l'intégration, on considère comme native une publicité en ligne qui se situe hors des emplacements habituels (bannières, ilot, gratte-ciel) en plus d'émuler l'ergonomie et le design du site qui l'accueille. Quant au contenu, la publicité native est traditionnellement arrimée à la ligne éditoriale du site hôte ou aux intérêts et comportements de l'usager visé.

Dans cette première partie, nous tentons donc de cerner les contours de l'écosystème de la publicité native en proposant deux catégories qui semblent faire consensus (IAB, 2013; IAB France, 2014; Manic, 2015): les écosystèmes intégré et périphérique. Nous répertorions six formes typiques réparties en deux grandes catégories selon qu'elles s'intègrent ou non au contenu informationnel du support. Précisons que nous n'avons tenu compte que des formats pouvant se retrouver sur des sites d'information, excluant d'emblée un ensemble protéiforme de contenus pouvant parfois être qualifiés de PN comme les publicités sur YouTube ou même les wikis (Henrard \& Pierra, 2015).

\section{2. Écosystème intégré}

La première catégorie de publicité native peut être qualifiée d'intégrée (voir tableau 1), car elle s'insère directement au sein du flux informationnel du support où les publicités sont diffusées. Dans un premier cas, cette intégration est sociale puisque la PN prend la forme des contenus générés par les usagers du site. C'est le cas des " posts sponsorisés » sur les principaux médias socio numériques. L'aspect natif est alors essentiellement ergonomique puisque les contenus diffèrent sensiblement de ceux générés par les usagers quant à leurs finalités. Une seconde forme de $\mathrm{PN}$ se révèle davantage éditoriale en imitant les thématiques du support. Les articles et dossiers publiés dans les médias d'information relèvent de cette catégorie. Si l'aspect natif touche aussi à l'ergonomie, la plupart des PN ressemblant formellement aux articles, il n'est pas rare que celle-ci prenne la forme d'un dossier à la mise en forme plus ambitieuse, mêlant différents formats sémiotiques au sein d'un dispositif riche. Enfin, les liens sponsorisés sont aussi évoqués comme forme de PN. Cette dernière catégorie jouit d'une forte visibilité au sein des professionnels davantage intéressés par l'automatisation des achats d'espaces rendue possible par les enchères en ligne. Elle est telle que le livre blanc de l'Interactive Advertising Bureau destiné à fournir une ébauche de normalisation a provoqué une polémique au sein du monde des publicitaires, déplorant la réduction des potentialités de la PN à cette dimension. L'intégration est ici à la fois ergonomique et éditoriale et de 
nombreuses interventions légales ont dû être effectuées pour clarifier le caractère commercial de ces liens (Seligman, 2015).

Ajoutons que ces publicités intègrent systématiquement une possibilité d'interactivité par le clic, qui peut renvoyer vers des contenus endogènes ou exogènes au site hôte. Si les PN sociales peuvent renvoyer indifféremment vers les deux, précisons que la vocation des PN éditoriales consiste davantage à conserver l'internaute au sein de leurs contenus tandis que les liens sponsorisés renvoient systématiquement vers des sites tiers.

\begin{tabular}{|c|l|l|l|l|}
\hline \multicolumn{2}{|c|}{ Flux } & \multicolumn{1}{c|}{$\begin{array}{c}\text { Contenu } \\
\text { endogène }\end{array}$} & \multicolumn{1}{c|}{$\begin{array}{c}\text { Contenu } \\
\text { exogène }\end{array}$} & Exemple \\
\hline Social & $\begin{array}{c}\text { Vidéo, photo, } \\
\text { texte. } \\
\text { Ergonomie } \\
\text { identique au site } \\
\text { hôte. }\end{array}$ & $\begin{array}{c}\text { Lien vers } \\
\text { section dédiée } \\
\text { (ex. Page FB) }\end{array}$ & $\begin{array}{c}\text { Aguiche } \\
\text { vers un site } \\
\text { externe (site } \\
\text { de } \\
\text { l'annonceur) }\end{array}$ & Annexe 1 \\
\hline Éditorial & $\begin{array}{c}\text { Texte } \\
\text { s'apparentant à un } \\
\text { article de l'éditeur. } \\
\text { Ergonomie et } \\
\text { design identiques. }\end{array}$ & $\begin{array}{l}\text { Lien vers } \\
\text { article } \\
\text { sponsorisé. } \\
\text { Lien vers } \\
\text { section } \\
\text { dédiée. }\end{array}$ & $\begin{array}{l}\text { Plus } \\
\text { rarement, un } \\
\text { lien vers } \\
\text { l'annonceur. }\end{array}$ & Annexe 2 \\
\hline Liens & $\begin{array}{c}\text { Suggestions } \\
\text { sponsorisés } \\
\text { sponsorisées } \\
\text { intégrées au flux, } \\
\text { avec mention. } \\
\text { Ergonomie } \\
\text { similaire. }\end{array}$ & S/O & $\begin{array}{c}\text { Mots clés } \\
\text { vers le site de } \\
\text { l'annonceur }\end{array}$ & Annexe 3 \\
& \multicolumn{2}{|l}{} & \\
\hline
\end{tabular}

\section{Tableau 1 : formes de PN intégrées}

\section{3. Écosystème périphérique}

La seconde catégorie de PN conserve des caractéristiques qui permettent de les distinguer des contenus propres au support (voir tableau 2). À ce titre, elles diffèrent moins des formats publicitaires traditionnels. L'aspect natif tient cependant à l'adaptation du fond au contenu des pages. Ainsi, les listes promues concernent spécifiquement les contextes d'achat. Elles ajoutent un ensemble de produits similaires sous un produit consulté. Elles peuvent se réduire à une présentation du produit ou intégrer des éléments de comparaison, d'évaluation, etc. Dans une forme 
proche, mais pouvant toucher des contextes de navigation plus larges, les modules de recommandation constituent aussi des espaces identifiés comme complémentaires au contenu diffusé par le support, qui proposent des liens diversifiés en lien avec ce dernier. Dans sa version la plus proche des formats traditionnels, la PN peut aussi se révéler un simple contenu contextuel inséré dans un espace publicitaire traditionnel.

Ces formes prévoient elles aussi l'interaction par clic en renvoyant alors vers des contenus endogènes ou exogènes. $\mathrm{Si}$ les publicités standards renvoient traditionnellement vers des sites tiers, les deux autres formes peuvent abriter indifféremment des liens endogènes ou exogènes.

\begin{tabular}{|c|c|c|c|c|}
\hline & Apparence & $\begin{array}{l}\text { Contenu } \\
\text { endogène }\end{array}$ & $\begin{array}{l}\text { Contenu } \\
\text { exogène }\end{array}$ & Exemple \\
\hline $\begin{array}{c}\text { Liste } \\
\text { promue }\end{array}$ & $\begin{array}{l}\text { Liste de comparaison } \\
\text { de produits, de prix, de } \\
\text { caractéristiques. } \\
\text { Commentaires ou } \\
\text { analyses d'apparence } \\
\text { journalistique. }\end{array}$ & $\begin{array}{l}\text { Lien vers } \\
\text { une section } \\
\text { dédiée au } \\
\text { produit }\end{array}$ & \begin{tabular}{l}
\multicolumn{1}{c}{ Lien } \\
vers site \\
des \\
produits \\
comparés
\end{tabular} & Annexe 4 \\
\hline $\begin{array}{l}\text { Module } \\
\text { de } \\
\text { recommand } \\
\text { ation } \\
\end{array}$ & $\begin{array}{l}\text { Module en marge du } \\
\text { site suggérant des } \\
\text { articles liés au contenu } \\
\text { affiché }\end{array}$ & $\begin{array}{l}\quad \text { Liens } \\
\text { vers section } \\
\text { dédiée du } \\
\text { site }\end{array}$ & $\begin{array}{l}\text { Liens } \\
\text { vers un } \\
\text { autre site }\end{array}$ & Annexe 5 \\
\hline $\begin{array}{l}\text { Publicité } \\
\text { standard } \\
\text { avec } \\
\text { contenu }\end{array}$ & $\begin{array}{l}\text { Espace publicitaire } \\
\text { traditionnel avec } \\
\text { contenu lié au contenu } \\
\text { de la page }\end{array}$ & $\mathrm{S} / \mathrm{O}$ & $\begin{array}{l}\text { Liens } \\
\text { vers un } \\
\text { autre site }\end{array}$ & Rare \\
\hline
\end{tabular}

\section{Tableau 2: formes de PN périphériques}

En somme, on le constate, ce qui définit et semble émerger de cette typologie réside dans l'émulation du contenu et du design de cette forme publicitaire. D'autant que dans plusieurs cas, l'usager reste à l'intérieur du site hôte. Le risque de confusion est alors important : est-ce que le contenu est bien celui d'un annonceur tiers ou du site hôte qu'il a l'habitude de consulter ? Ainsi, bien que normalement la publicité native est assortie d'une mention (ex. «sponsorisée par»), d'une différenciation visuelle (ex. un cadre de couleur différent) ou d'une désignation de 
l'annonceur (ex. logo de l'annonceur qui commandite la publicité), cette émulation demeure problématique pour les professionnels de l'information et du marketing ${ }^{1}$.

\section{Discours professionnels autour de la PN}

Afin de comprendre comment les professionnels de l'information et du marketing se saisissent de l'impact de la PN sur leur travail et leur industrie, nous avons procédé à une collecte des discours entourant ces enjeux entre le 11 mai $2015^{2}$ et le 21 avril 2016 sur six sites francophones :

- Infopresse.com (Québec)

- Stratégies.fr (France)

- Grenier aux nouvelles (Québec)

- Assises du journalisme (France)

- Fédération professionnelle des journalistes (Québec)

- Syndicat national des journalistes (France)

À l'aide des moteurs de recherche de chaque site, nous avons répertorié tous les articles traitant de «publicité native», «native advertising», "marketing de contenu $»^{3}$ et « content marketing». En tout 250 articles ont été dépouillés. Nous avons conservé 228 passages traitant de la relation entre publicité et information dans le contexte de la publicité native.

Par la suite, pour chacun des articles, nous avons identifié, de façon inductive, les rationalités sous-jacentes concernant l'utilisation de la PN (Paillé \& Mucchielli, 2012). Dit autrement, sous quels motifs peut-on (doit-on) utiliser ou non la PN. Au final, 116 rationalités ont été déterminées.

Finalement, nous avons catégorisé ces rationalités selon « les relations qui existent entre les faits explicites et le sens implicite du discours des gens » (Attride-Stirling, 2001, p. 387. Nous traduisons).

Ainsi, nous avons circonscrit six catégories de rationalités issues du discours des professionnels du marketing, de la publicité et du numérique (Figure 1). Les nombres sur chaque barre représentent le nombre de passages traitant de cette rationalité.

\footnotetext{
${ }^{1}$ Les enquêtes soulignent que les PN sont d'ailleurs très mal identifiées par leurs lecteurs : http://www.infopresse.com/dossier/2014/11/20/publicite-native-tour-d-horizon

${ }^{2}$ Le 11 mai 2015 marque la mise en route de Instant Articles, le module de promotion d'articles de médias d'information de Facebook. Ce lancement avait suscité une vague de réactions à propos des contours flous entre information et promotion, apparaissant donc pour nous comme un jalon important pour observer le discours des professionnels.

${ }^{3}$ Il apparait que certains auteurs et professionnels associent la publicité native au contenu de marque.
} 
La PN favorise l'engagement/une meilleure expérience

La PN permet d'être vu/d'atteindre notre cible

La PN permet de générer des revenus/meilleur retour sur..

La publicité doit maintenant faire partie du contenu

La PN est plus pertinente pour la cible

La PN doit être clairement identifiée

Il faut s'adapter aux intérêts de la cible

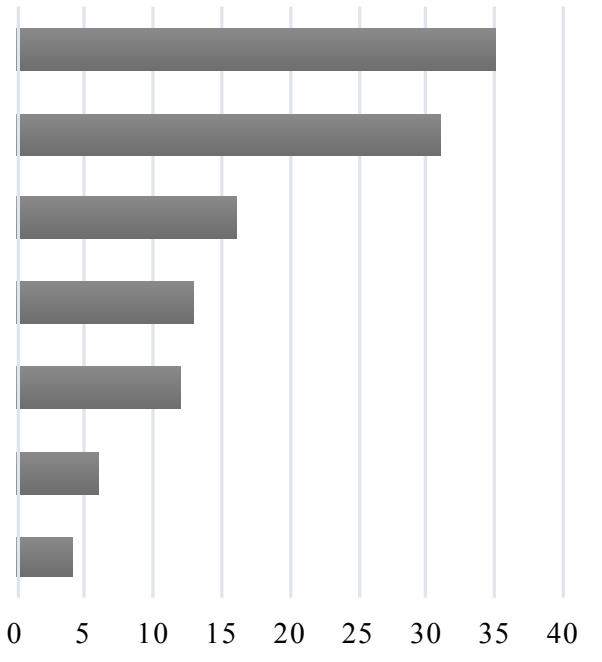

Figure 1: rationalités des professionnels du marketing, de la publicité et du numérique

Suivant la même procédure, nous avons circonscrit, du côté des professionnels de l'information, huit catégories de rationalités (Figure 2). 


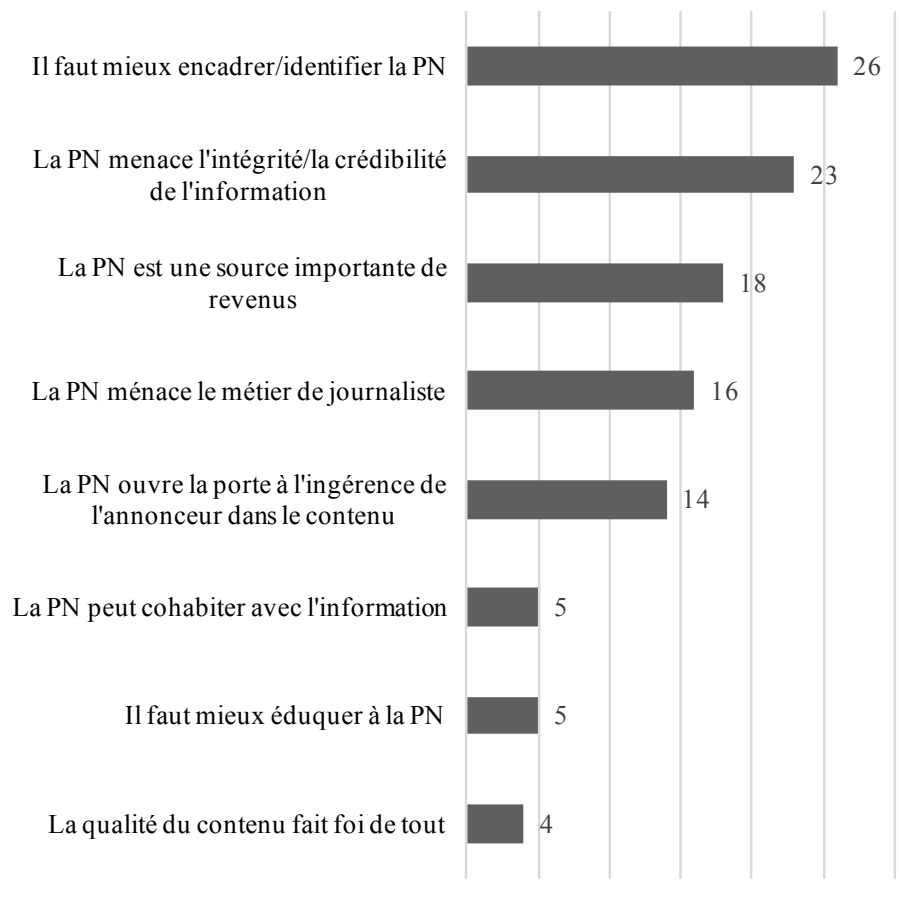

Figure 2 : catégories de rationalités des professionnels de l'information

\section{Analyse des discours professionnels autour de la PN}

Une fois appréhendés les discours des deux industries, ce qui nous importait davantage était de voir quelles étaient les zones de convergence ou de divergence dans les discours. Le but est ici de tracer un portrait du bouleversement éthique qu'a imposé la PN dans les deux univers.

Pour ce faire, nous avons placé sur une carte perceptuelle les discours selon les modalités suivantes (Felx, et al., 2014).

Nous avons d'abord considéré l'importance proportionnelle d'une catégorie de discours sur l'ensemble des discours des deux secteurs. Ce premier axe nous permettait de voir la prégnance d'un discours.

Nous avons ensuite voulu relever le degré de polarisation du discours. Dit autrement, à quel point un discours était exclusif à l'un des secteurs et à quel point certaines rationalités pouvaient être apparentées. Pour concevoir l'axe de 
polarisation, nous avons attribué un coefficient de convergence selon l'échelle suivante :

- -2 = polarisé strictement information (ex. «La PN menace le métier de journaliste)

- $\quad-1$ = légèrement polarisé information, mais avec un écho marketing (ex. « La $\mathrm{PN}$ est une source de revenus » peut s'apparenter à « La PN permet de générer des revenus/un meilleur retour sur investissement »)

- 1 = légèrement polarisé marketing, mais avec des échos en information (ex. « Générer des revenus/un meilleur retour sur investissement » peut s'apparenter à « La PN est une source de revenus »)

- 2 = polarisé strictement marketing (ex. La PN favorise l'engagement/une meilleure expérience)

Ce coefficient de convergence ne suffit toutefois pas à traduire à quel point les deux univers convergent ou divergent. De fait, que des discours se rapprochent dans leurs soubassements rationnels n'indique pas nécessairement que les arguments qu'ils charrient constitueraient un dispositif de compromis. Nous avons donc pondéré ce coefficient de convergence avec le poids de chaque discours. Ainsi un discours très important en information, mais avec des échos en marketing, se trouvera malgré tout plus polarisé vers l'information (ex. La PN est une source importante de revenu) qu'un discours à faible importance en information qui a des échos en marketing (ex. La qualité du contenu fait foi de tout), la logique étant qu'un discours peu galvaudé est moins porteur de ce qui importe au sein d'une communauté de parole, donc moins apte à la constituer (Cooren, 2013; Philipsen, 1992).

La figure suivante (Figure 3) montre le positionnement des catégories de rationalités. La taille des cercles permet de visualiser le poids des discours. Les cercles pâles correspondent aux discours marketing ; les cercles foncés, aux discours en information. 


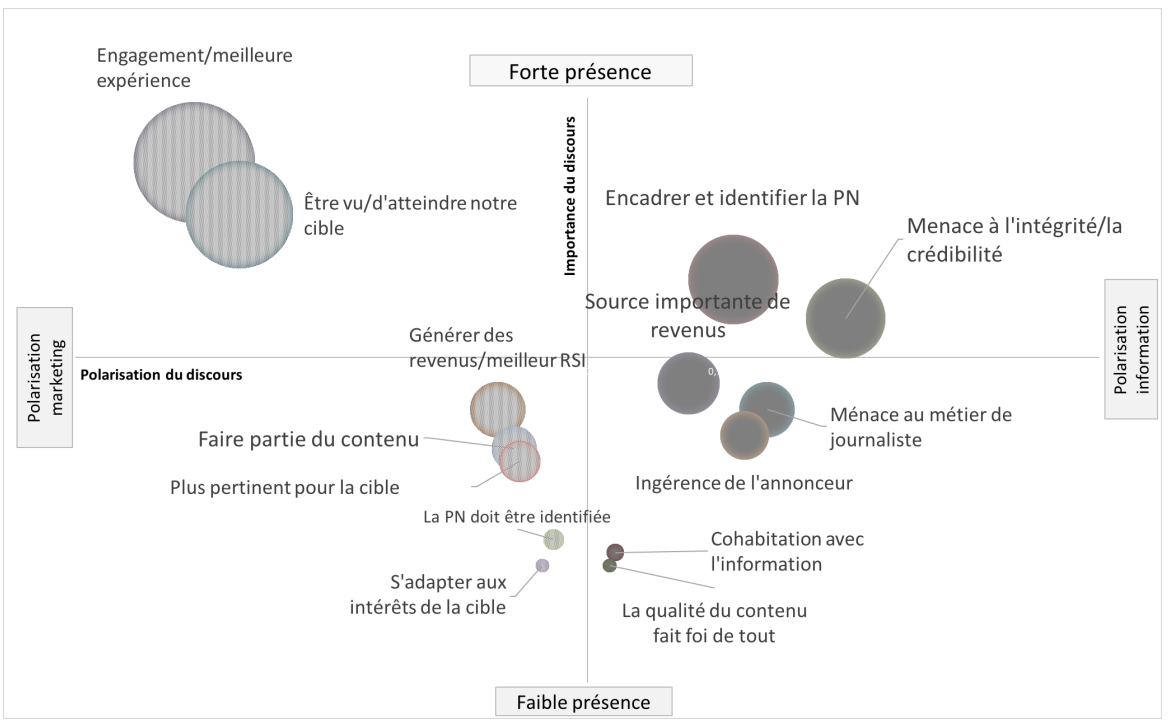

Figure 3 : mapping des rationalités

Nous procédons à une analyse critique de ce mappage infra. Pour l'instant, une dernière étape est nécessaire pour observer les tensions éthiques entre les discours et les codes déontiques.

\section{Que disent les codes et les chartes ?}

De façon à pouvoir adosser ces discours aux codes, chartes et guides déontiques, nous avons procédé à une recension des articles entourant la relation entre information et publicité en Europe et en Amérique du Nord. Aussi avons-nous scruté 18 codes régissant l'industrie de la publicité et de l'information. Au total, 80 articles traitaient de la relation entre publicité et information. Une fois de plus, nous avons catégorisé le contenu de ces codes (Figure 4). 
40 Professionnalisation et éthique de la communication

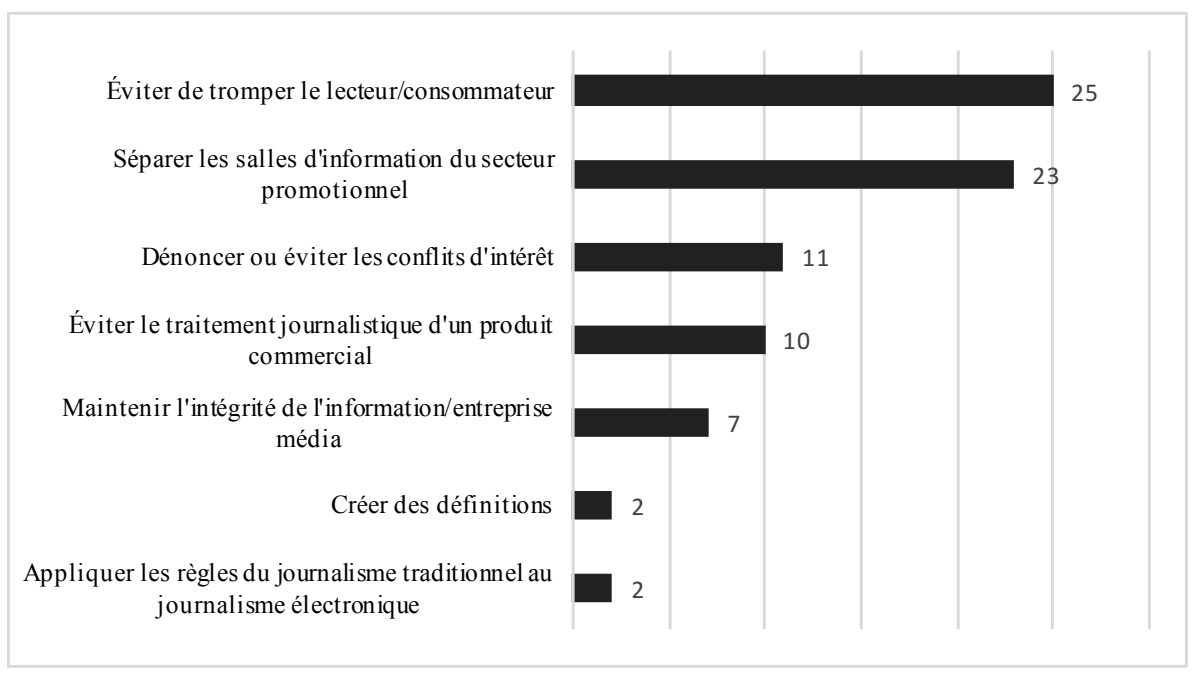

Figure 4 : catégorisation des articles entourant la gestion de la relation publicité et information

Avec à la fois des discours portant sur les raisons d'utiliser ou non la PN et les règles déontiques servant à encadrer la pratique, lorsque la publicité semble sortir de son cadre de référence habituel, nous pouvons maintenant mieux comprendre le bouleversement professionnel engendré par l'arrivée massive de la PN.

\section{Discussion : convergences et divergences éthiques}

\subsection{Divergences idéologiques inconciliables}

Le mappage nous permet d'emblée de remarquer que les discours les plus prégnants sont ceux qui sont les plus polarisés. De fait, on remarque rapidement que le discours sur l'expérience et l'engagement de la cible propre au marketing va tout à fait dans le sens de la nécessité, pour les marques, d'être invités dans l'univers d'un consommateur réfractaire à la publicité et à l'attention fragmentée (Dahlén \& Edenius, 2007; Lanham, 2006). En émulant un contenu éditorial ou en créant du contenu près de la ligne éditoriale du site hôte, les annonceurs s'assureraient un intérêt plus marqué :

Que ce soit en desktop ou en mobile, il est également possible de se tourner vers la création de contenu ou de contenu commandité, la publicité native, les relations publiques 2.0, etc. Une chose est claire : la pression est de plus en plus forte pour 
trouver des technologies publicitaires qui améliorent l'expérience usager et qui dissuadent les internautes à télécharger un dispositif d'adblocking ${ }^{4}$.

Cette expérience usager est soutenue par un désir de ne pas interrompre l'expérience en ligne et ainsi mieux atteindre les cibles, deuxième rationalité la plus forte pour les professionnels du marketing.

Or ce désir de ne pas contrevenir à l'expérience utilisateur est justement ce qui semble irriter les professionnels de l'information. On retrouve en effet à l'autre extrémité du spectre le fait que la PN menace l'intégrité et la crédibilité de l'information. De fait, pour les gens de l'information, l'interruption est justement ce qui permet de différencier les contenus publicitaires des contenus éditoriaux. Le fait de saboter cette distinction compromet l'intégrité du contenu.

Mais jusqu'à quel point cela [l'importance de la crédibilité] est-il compris, dans le public et parmi les annonceurs potentiels ? Pas trop, si on se fie au nombre de fois où des journalistes et des rédacteurs en chef se font demander : "Combien ça coûte, un reportage chez vous? $»^{5}$

Et, dégât collatéral, sur l'imprimé ou sur le web, la perte de confiance due à la publicité camouflée retombera sur les vrais articles d'information. Le lecteur se dira «Si ce papier camoufle de la pub, pourquoi un autre serait-il davantage digne de confiance ? » Tous les journalistes pâtiront de ces pratiques. ${ }^{6}$

On voit d'ailleurs l'enjeu éthique : les médias ont des audiences qui cherchent du contenu de qualité, audiences qu'ils peuvent par la suite offrir aux annonceurs. Or si la qualité de ce contenu est amalgamée avec du contenu télescopé par les marques, quid de la valeur de ce contenu ? La tension est bien illustrée théoriquement par la définition de la compétence communicationnelle chez Quéré (1982). Celle-ci suppose en effet la réunion de quatre principes : l'intelligence réciproque (prétention à l'intelligibilité), le partage de connaissances (prétention à la vérité), la confiance réciproque (prétention à la sincérité) et l'entente réciproque sur le type de situation. Quelles que soient la qualité et la véracité des contenus diffusés, les deux derniers points soulignent que leur interprétation repose tout autant sur la relation établie entre les interlocuteurs. La justification de la PN par la qualité des contenus ne lève donc qu'une part de ces enjeux communicationnels, que les travaux sur l'énonciation ont bien identifiée comme relative (Veron, 1985).

Nous nous trouvons donc dans des divergences idéologiques opposant deux éthiques: fournir une expérience non intrusive et engageante pour atteindre une cible réfractaire à la publicité et maintenir l'intégrité du contenu en séparant

\footnotetext{
${ }^{4} \mathrm{http}: / /$ www.infopresse.com/article/2015/8/10/adblocking-qu-est-ce-que-1-avenir-nous-reserve

${ }^{5} \mathrm{http}: / /$ www.fpjq.org/realite-medias-101-apprentissages-a-dure/

${ }^{6} \mathrm{http}: / /$ snjexpress.hautetfort.com/archive/2014/07/12/les-habits-neufs-du-publiredactionnel5409049.html
} 
publicité et promotion. Plus fondamentalement, c'est donc une divergence entre focalisation sur l'énoncé et prise en compte de l'énonciation qui est mise en jeu.

Qui plus est, la force de cette divergence montre à quel point les codes et chartes ne suffisent pas à encadrer la PN. De fait, alors que l'obligation de ne pas tromper le consommateur et la nécessité de séparer information et marketing constituent les injonctions éthiques les plus fortement représentées dans l'appareillage déontique (voir Erreur ! Nous n'avons pas trouvé la source du renvoi. supra), ce sont ces mêmes codes qui sont mis à mal par ces divergences. Au nom d'une expérience et d'un engagement améliorés, les professionnels du marketing ne voient pas de problème dans la $\mathrm{PN}$, se rabattant sur le fait «que la PN doit être bien identifiée », comme le montre une des rationalités évoquées tant qu'elle est bien identifiée. Or ce discours n'est pas très prégnant chez les gens du marketing - seulement six entrées sur les 228 - ce qui donne à penser que l'identification n'est pas un problème pour ceux-ci. D'autant que, comme le démontre la section suivante, une rationalité structurelle, vient justifier, de part et d'autre, l'utilisation de la PN.

\subsection{Rapprochements structurels}

Une zone de convergence se dessine autour de rationalités «structurelles ", à savoir de la nécessité économique de monétiser le contenu des plateformes d'une part et, d'autre part, de favoriser un meilleur retour sur investissement. Ainsi, générer le second trouve un écho du côté de l'information, la PN étant vue comme une source de revenus importante:

« Le seul secteur de revenus en croissance, c'est la publicité native $[\ldots]$ on crache là-dessus, on se tire dans le pied. » Éric Trottier, éditeur-adjoint du journal La Presse ${ }^{7}$.

Or cette cohabitation par nécessité économique engendre des effets collatéraux qui menacent le métier de journaliste. De fait, par souci de monétiser son contenu - mais également parce que les journalistes font face à la précarisation de leur emploi - les journalistes doivent parfois brader leurs expertises pour produire des publicités natives de type « flux intégré - éditorial» :

Depuis que j'ai quitté Les Affaires [une revue économique], je ne suis pas la seule à me demander si je ferais le même métier de journaliste que je défends farouchement depuis 30 ans, ou si je devrai dorénavant m'identifier comme " productrice de contenu ». Suzanne Dansereau, journaliste indépendante ${ }^{8}$

Ce qui touche directement l'identité des journalistes et leur appartenance professionnelle :

« [...] les journalistes devront choisir leur camp. Ceux qui font de la publicité native ou du marketing de contenu devraient être bannis de la [Fédération

\footnotetext{
${ }^{7} \mathrm{http}: / /$ www.fpjq.org/on-ne-cracher-dessus/

${ }^{8} \mathrm{http}: / / \mathrm{www}$. fpjq.org/collision-frontale/
} 
professionnelle des journalistes du Québec] et interdits de pige dans les sections régulières du journal », Charles Côté, président du Syndicat des travailleurs de 1'information du journal La Presse (STIP) ${ }^{9}$.

On voit d'ailleurs, dans la proximité des rationalités « La PN menace le métier de journaliste » et « La PN favorise l'ingérence de l'annonceur », deux préoccupations qui ne font pas partie des considérations des professionnels du marketing. De la même façon, en termes de prégnance des discours, la PN, comme source de revenus, côtoie la crainte d'une perte d'intégrité journalistique. C'est dire que les questions économiques, sans surprise, pèsent lourdement sur les considérations éthiques.

\subsection{Convergences sur fond de contenu}

Finalement, nous observons une zone de convergence dans les discours, mais cette fois-ci sur fond d'un consommateur intelligent qui souhaite d'abord du contenu de qualité. De fait, alors que les consommateurs chercheraient du contenu pertinent, riche et arrimé à ses intérêts, une cohabitation avec l'information serait possible sans porter atteinte à l'intégrité de l'information :

Mais pour le reste, le « native advertising » ne mérite pas tant de méfiance : «J'ai confiance en l'intelligence des uns et des autres. Les gens ne sont pas bêtes. Le risque, ce serait si tout le monde devient idiot... ", Guillaume Sire, maître de conférence spécialisé dans le native advertising en entrevue aux Assises du journalisme ${ }^{10}$.

C'est sur fond d'une qualité de contenu que cette convergence serait possible : Le succès de ces articles sponsorisés permet aux médias d'être pleins d'espoir. D'autant que le trafic n'y est pas moins important que sur le reste des contenus, bien au contraire. C'est l'intérêt de l'article qui fait venir ou non le lecteur. « Au Figaro on veut apporter quelque chose de qualité à nos utilisateurs [...] Et le fait qu'une marque soit partenaire ne rend pas un article moins intéressant », Baptiste Huriez, directeur adjoint marketing, Figaro ${ }^{11}$.

L'argument de l'intelligence du lecteur fait sourire face aux résultats d'enquête cités supra et témoignant d'une faible identification des PN. Il constitue d'ailleurs l'un des arguments recensés par Drumwright and Murphy (2004) parmi les raisons de la myopie des publicitaires. Remettre la responsabilité sur l'intelligence du public constitue effectivement un moyen régulièrement employé par les publicitaires pour se dédouaner de leurs responsabilités.

Les dispositifs d'identification des PN seraient le garde-fou permettant de ne pas entacher l'intelligence du lecteur. Or ceci semble appeler un rapprochement en production entre les journalistes et les producteurs de contenu : comment, de fait, garantir un « article intéressant » dans un environnement éditorial, donc en émuler la

\footnotetext{
${ }^{9}$ http://www.fpjq.org/collision-frontale/

${ }^{10} \mathrm{http}$ ://assises.journalisme.epjt.fr/enquete-native-advertising-les-liaisons-dangereuses

${ }^{11} \mathrm{http}$ ://assises.journalisme.epjt.fr/enquete-native-advertising-les-liaisons-dangereuses
} 
qualité et la profondeur, si ce n'est que par l'embauche de journalistes. Les codes et chartes ont pour vocation de garantir l'imperméabilité entre les salles de nouvelles et celle des ventes, d'éviter les conflits d'intérêts et le traitement journalistique d'une nouvelle commerciale. Quid d'une nouvelle justement construite suivant l'ingérence du commercial dans l'information, tout en respectant la « profondeur » du traitement journalistique ? Il y a là une perte de repères sur la permanence et la cohérence des positions, qui complète la myopie morale en lui ajoutant une part de mutisme : Drumwright et Murphy (2004) identifient effectivement une tendance à compartimenter les dimensions d'un problème afin d'éviter d'en identifier les contradictions. Refuser d'aborder les enjeux associés à la $\mathrm{PN}$ selon les points soulevés par les autres acteurs impliqués et interrompre le raisonnement afin de ne pas en souligner les contradictions se renforcent effectivement pour expulser les enjeux éthiques des réflexions des publicitaires.

\section{Conclusions : des divergences fondamentales, des convergences fragiles et un métier à réinventer}

Par cette recherche exploratoire, nous avons tenté d'observer la façon dont la publicité native agit comme source de tensions et bouscule les repères professionnels des gens de marketing comme de ceux de l'information. En relevant les différentes rationalités entourant l'utilisation de la PN, nous croyons pouvoir mieux saisir ces bouleversements.

D'une part, nous avons démontré des tensions inconciliables. D'abord, les éditeurs tentent de maintenir une « bienveillance » (Cossette, 2009) vis-à-vis de leur lectorat, bienveillance qui peut être entachée par une information qui n'est plus portée par la nécessité d'informer et hors de toute ingérence. Ensuite, les marqueteurs sont en quête d'attention, avec une cible qui demanderait, selon eux, du contenu plus pertinent, plus riche, plus engageant, et non de la publicité intrusive.

C'est dans cette recherche mutuelle d'un lectorat/consommateur fuyant - dont la désaffection menace les deux industries - que des convergences structurelles pointent : monétisation de l'expertise en contenu pour les supports médias ; meilleur retour sur investissement pour les marketeurs; attraction d'un lectorat par du contenu commandité. Cette convergence a pour effet, sous la force des rationalités sur l'intégrité de l'information et du métier de journaliste, de faire apparaître un nouveau métier hybride: le producteur de contenu. Parfois associé aux médias comme pigiste ou salarié -, mais dont l'appartenance professionnelle ne semble pas faire l'unanimité, ce nouveau professionnel du contenu émanerait donc d'un double rejet. D'abord, le professionnel du journalisme qui, faute d'emploi ou par cause de précarité, se tourne vers cette manne financière, tout en risquant l'exclusion professionnelle. Puis, le professionnel du marketing, dont la production « traditionnelle» n'est plus à même d'intéresser un public cible réfractaire à ses 
charmes, doit émuler les codes d'une autre discipline. A fortiori, dans un contexte où les médias d'information condamnent vigoureusement, à l'instar des gens de marketing, l'utilisation des logiciels de blocage qui frustrent les éditeurs de revenus substantiels (Heine, 2016).

Or toute la fragilité de cette convergence possible - illustrée par la faible importance des rationalités entourant la qualité des contenus - porte à croire que ce producteur de contenu sera persona non grata chez les professionnels de l'information. Reste à voir la place qu'il pourra occuper dans la chaîne de production marketing. Et surtout, à quel genre de codes, de charte ou à quels aménagements déontiques il obligera.

Cette recherche se veut un point de départ pour appréhender un phénomène dont la portée professionnelle nous échappe encore. Certes, pour l'heure, le corpus restreint géographiquement et professionnellement nous invite à creuser davantage ce phénomène pour le comprendre plus finement. Qui plus est, bien que cette première exploration semble reproduire un stéréotypage quelque peu manichéen -le journaliste drapé de vertu et le marketeur avide - nous croyons que la réalité organisationnelle admette plus de zones grises que le laisse présager nos données. Malgré tout, il nous apparaît intéressant déjà d'ouvrir trois pistes pour continuer l'approfondissement : observer les identités et formes que prennent ces producteurs de contenu ; mieux comprendre les dynamiques organisationnelles se jouant dans les organisations médiatiques pour les accueillir (ou non). À l'autre bout du spectre, nous croyons qu'il devient impératif également d'étudier les usages autour de la PN : le lecteur est-il à ce point berné ? est-il en effet simplement à la recherche de « contenu engageant» qu'importe sa source et sa rigueur ? perçoit-il les mécanismes d'identification prônés?

Les modèles d'affaires des médias d'information, la montée des logiciels de blocage publicitaire qui ne semble pas s'essouffler : tout porte à croire que le bouleversement engendré par la PN contribuera encore à ces chevauchements de genre, renverra les professionnels à leur éthique et confrontera les codes, chartes et guides déontologiques dans leurs limites. 


\section{Bibliographie}

Attride-Stirling, J. (2001). Thematic networks: an analytic tool for qualitative research. Qualitative Research, 1(3), 385-405. doi:10.1177/146879410100100307

Cooren, F. (2013). Manières de faire parler : Interaction et ventriloquie. Lormont: Éditions du Bord de l'Eau.

Cossette, C. (2009). Ethique et publicité. Québec: PUL.

Dahlén, M. \& Edenius, M. (2007). When Is Advertising Advertising? Comparing Responses to Non-Traditional and Traditional Advertising Media. Journal of current issues and research in advertising, 29(1), 33-42.

Devèze, J. (2005). La prolifération des chartes éthiques et des codes de déontologie dans les domaines professionnels relevant des sciences de l'information et de la communication: éléments descriptifs. In S. Agostinelli (Ed.), L'éthique des situations de communication numérique. Paris: Harmattan.

Dienel, E. \& Robinson, J. (2015). Transparency, Purpose, and the Empowered Consumer: A New Paradigm for Advertising. Récupéré à http://www.bsr.org/reports/BSR_Participant_Responsibility_Transparency_in_ Advertising_March_2015.pdf

Drohan, M. (2016). Le journalisme de qualité a-t-il un avenir au Canada? Récupéré http://www.ppforum.ca/sites/default/files/PM\%20Fellow_March_11_FR_1.pdf

Drumwright, M. E. \& Murphy, P. E. (2004). How advertising practitioners view ethics: Moral muteness, moral myopia, and moral imagination. Journal of Advertising, 33(2), 7-24. http://www.jstor.org/stable/4189254

Eveno, P. (2004). Médias et publicité : une association équivoque mais indispensable. Le Temps des médias, 2(1), 17-27. doi:10.3917/tdm.002.0017

Felx, A., Kane, M., Corbière, M. \& Lesage, A. (2014). La cartographie des concepts. Une représentation visuelle et spatiale pour décrire les ressources résidentielles en santé mentale. In N. Larivière \& M. Corbière (Eds.), Méthodes Qualitatives, Quantitatives et Mixtes: Dans la Recherche en Sciences Humaines, Sociales et de la Santé (p. chapitre 23). Montréal: Presses de l'Université du Québec.

Floris, B. (2003). Espace public et sphère économique. Hermès, La Revue, 2, 127 136. http://www.cairn.info/revue-hermes-la-revue-2003-2-page-127.htm 
Heine, C. (2016). IAB Chief Blasts Adblock Plus as an "Immoral, Mendacious Coven of Techie Wannabes". Récupéré à http://www.adweek.com/news/technology/iab-chief-blasts-adblock-plusimmoral-mendacious-coven-techie-wannabes-169194

Henrard, P. \& Pierra, P. (2015). Guide du marketing de contenu. Montréal: Infopresse.

IAB. (2013). IAB Native Advertising Playbook. Récupéré à https://www.iab.com/wp-content/uploads/2015/06/IAB-Native-AdvertisingPlaybook2.pdf

IAB France. (2014). Le livre blanc du native advertising. Récupéré à http://www.iabfrance.com/system/files/nativeadvertising_a5.pdf?download=1

Laczniak, G. R. (1993). Marketing ethics: Onward toward greater expectations. Journal of Public Policy \& Marketing, 91-96. http://www.jstor.org/stable/30000114

Lanham, R. A. (2006). The economics of attention: Style and substance in the age of information. Chicago: University of Chicago Press.

Le Goff, D., Lefebvre, M.-J. \& Si Ammour, D. (2014). Native advertising : qui fait quoi dans les médias. Récupéré à http://www.strategies.fr/etudestendances/tendances/242960W/native-advertising-qui-fait-quoi-dans-lesmedias.html

Lefebvre, M.-J. (2014). Native ads: quand les journalistes se transforment en communicants. Récupéré à http://www.strategies.fr/etudestendances/tendances/238780W/native-ads-quand-les-journalistes-setransforment-en-communicants.html

Manic, M. (2015). The Rise of native advertising. Bulletin of the Transilvania University of Brasov. Economic Sciences. Series $V, 8(1)$, 53-58. http://webbut.unitbv.ro/bulletin/Series V/BULETIN I PDF/07_Manic_M.pdf

Martin, L. (2004). La « mauvaise publicité »: Sens et contresens d'une censure. Le Temps des médias, 2(1), 151-162. doi:10.3917/tdm.002.0151

Moumouni, C. (2005). Quand la publicité ressemble à l'information. Les dessous historiques et juridiques d'un glissement identitaire. In M.-F. Bernier, F. Demers, A. Lavigne, C. Moumouni \& T. Watine (Eds.), Pratiques novatrices en communication publique. Journalisme, relations publiques et publicité (pp. 127157). Québec: Presses de l’Université Laval.

Newman, N., Levy, David A \& Nielsen, R. K. (2015). Reuters Institute Digital News Report 2015. Récupéré à http://bit.ly/digitalnews2015 
Paillé, P. \& Mucchielli, A. (2012). L'analyse qualitative en sciences humaines et sociales (Sciences humaines \& sociales) (French Edition). https://www.amazon.ca/dp/B00AHD09HO

Palmer, M. (2004). Information et publicité : les « liaisons dangereuses »: Le cas des agences de presse. Le Temps des médias, 2(1), 41. doi:10.3917/tdm.002.0041

Philipsen, G. (1992). Speaking culturally: Explorations in social communication. Albany: State University of New York.

Quéré, L. (1982). Des miroirs équivoques. Aux origines de la communication moderne. Paris: Aubier.

Rosenwald, M. (2015). Digital news consumers unlikely to pay for content and increasingly block ads. Récupéré à http://www.cjr.org/analysis/reuters_digital_news_report.php

Saulnier, A. (2015). Des vendeurs du temple dans nos médias. Récupéré à $\mathrm{http}: / / w w w . f p j q . o r g / v e n d e u r s-t e m p l e-n o s-m e d i a s /$

Seligman, T. J. (2015). Native Advertising : The old is new again. The Computer \& Internet Lawyer, 32(7), 1-9.

Shields, M. (2016). Here's How Oversized Web Ads Are Encouraging Ad Blocking. Récupéré à http://www.wsj.com/articles/heres-how-oversized-web-ads-areencouraging-ad-blocking-1459159200

Shields, R. (2015). Confidence increases in native, but creative still a problem. Récupéré à http://www.thedrum.com/news/2015/07/14/confidence-increasesnative-creative-still-problem

Toussaint-Desmoulins, N. (2015). L'économie des médias (9e ed.). Paris: Presses universitaires de France.

Veron, E. (1985). L'analyse du "contrat de lecture": une nouvelle méthode pour les études de positionnement des supports presse. In IREP (Ed.), Les médias, expériences, recherches actuelles, applications (pp. 203-229).

WARC. (2016a). Agencies must avoid “irritation". Récupéré à http://www.warc.com/LatestNews/News/EmailNews.news?ID=36635

Warc. (2016b). Native to take half of digital display. Récupéré à www.warc.com/LatestNews/News/EmailNews.news?ID=36272

WARC. (2015). Marketers face 'digital backlash'. Récupéré à http://www.warc.com/News/Default.aspx?ID=35846

Wright, P. (1986). Schemer schema: consumers' intuitive theories about marketers' influence tactics. Advances in consumer research, 13(1), 1-3. 


\section{Annexe 1 : Exemple de publicité native « flux social »}

Dans l'exemple suivant, la publicité de « Levez la main pour le diabète » s'insère dans le flux social d'une page Facebook, s'arrimant ainsi à l'ergonomie, mais donnant également l'impression qu'il s'agit de L'actualité, un magazine d'affaires publiques, qui soutient la publication.

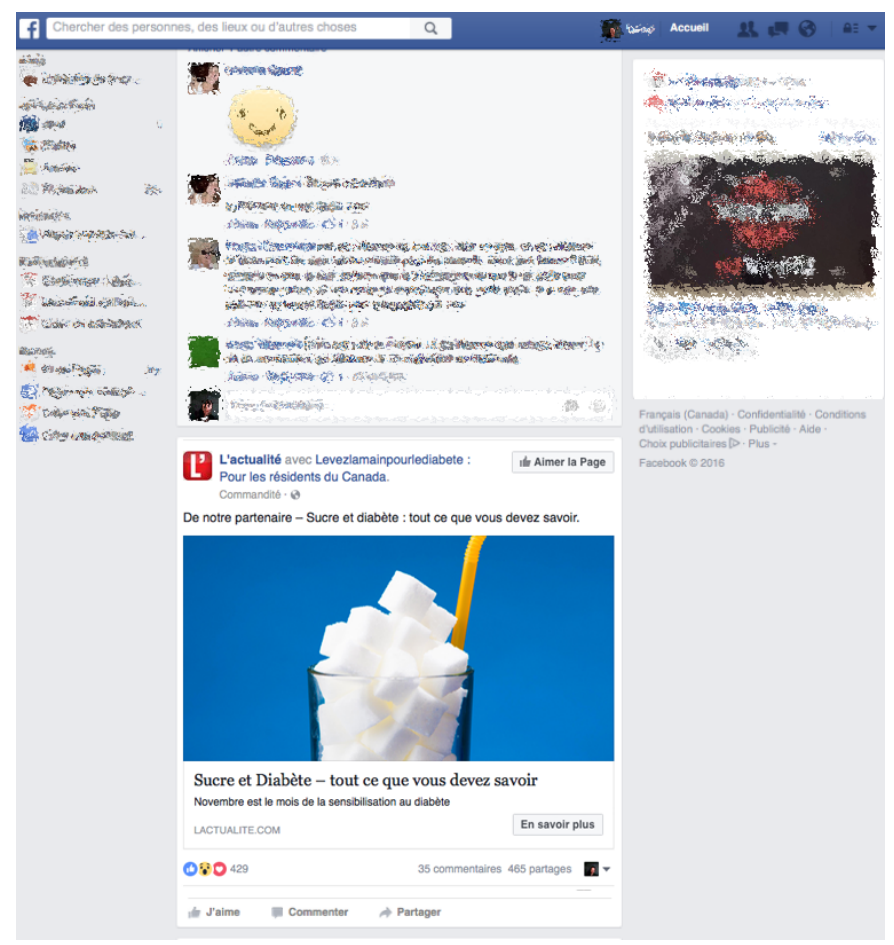




\section{Annexe 2 : Exemple de flux éditorial}

Dans cet exemple, les affaires, un magazine économique, propose une section appelée « Savoirs d'entreprise », qui se trouve à être constituée d'articles rédigés pour le compte d'annonceurs. Dans le cas ici, pour RDV Relève.

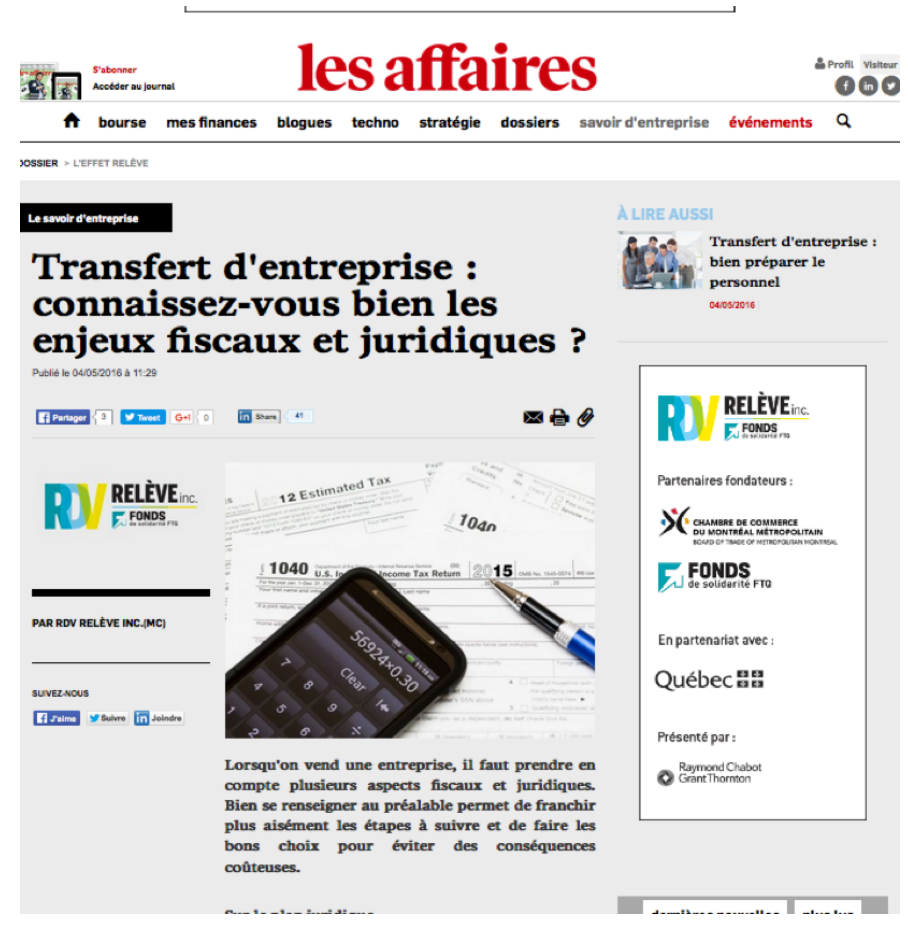


Confrontations, convergences éthiques entre marketing et information 51

\section{Annexe 3 : Exemple de liens sponsorisés}

Les liens marqués «Annonce », bien qu'émanant d'une recherche Google et s'insérant dans le flux d'autres liens, ont été payés.

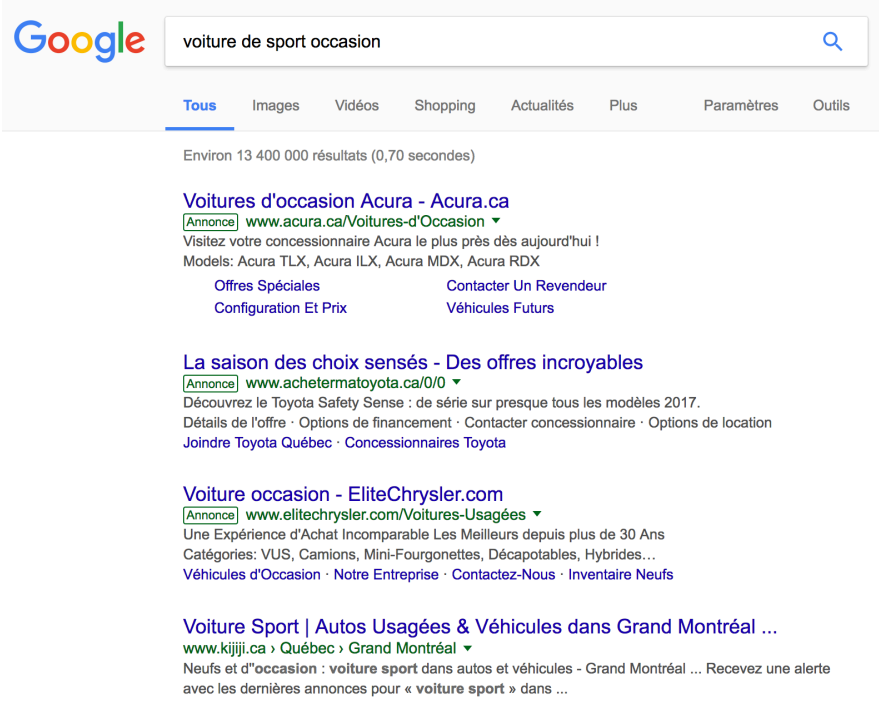




\section{Annexe 4 : Exemple de lien sponsorisé}

Sur le site d'EBay, entreprise de courtage et d'enchères en ligne, sont insérés, à même le flux d'ordinateurs d'occasion à vendre, des articles «promoted», c'est-àdire, qu'ils sont promus dans la liste.

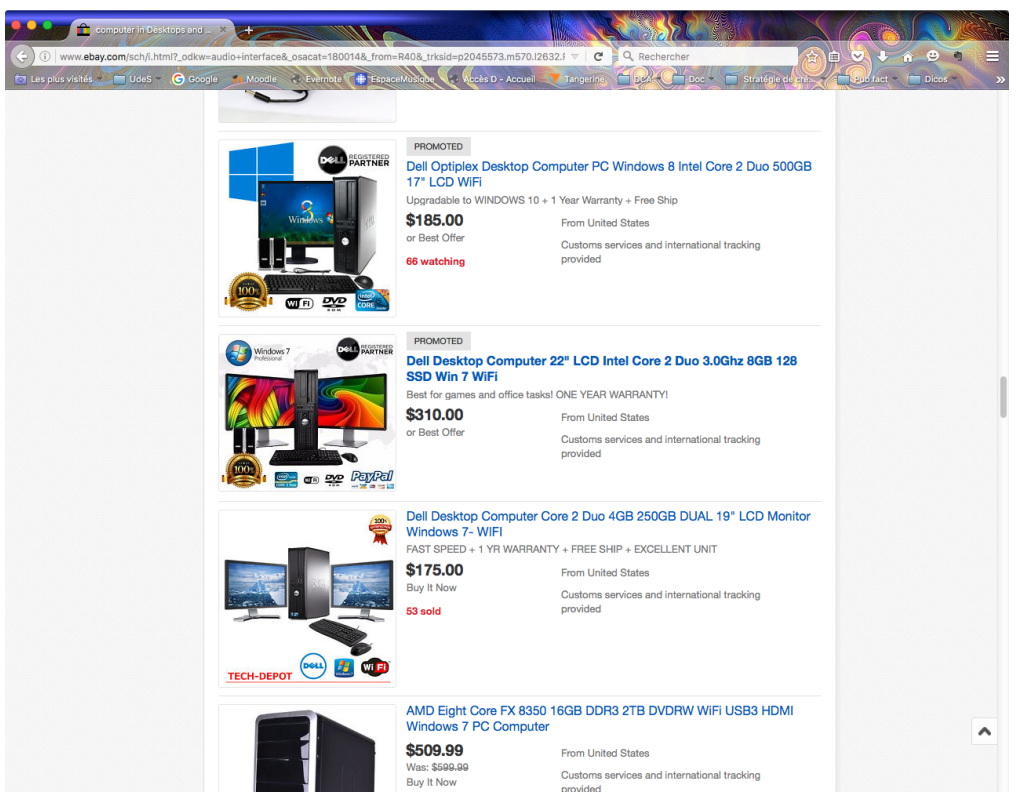




\section{Annexe 5 : Exemple de module de recommandation}

Dans cet exemple, des recommandations de machines à café apparaissent dans un bloc sponsorisé par le détaillant Linen Chest.

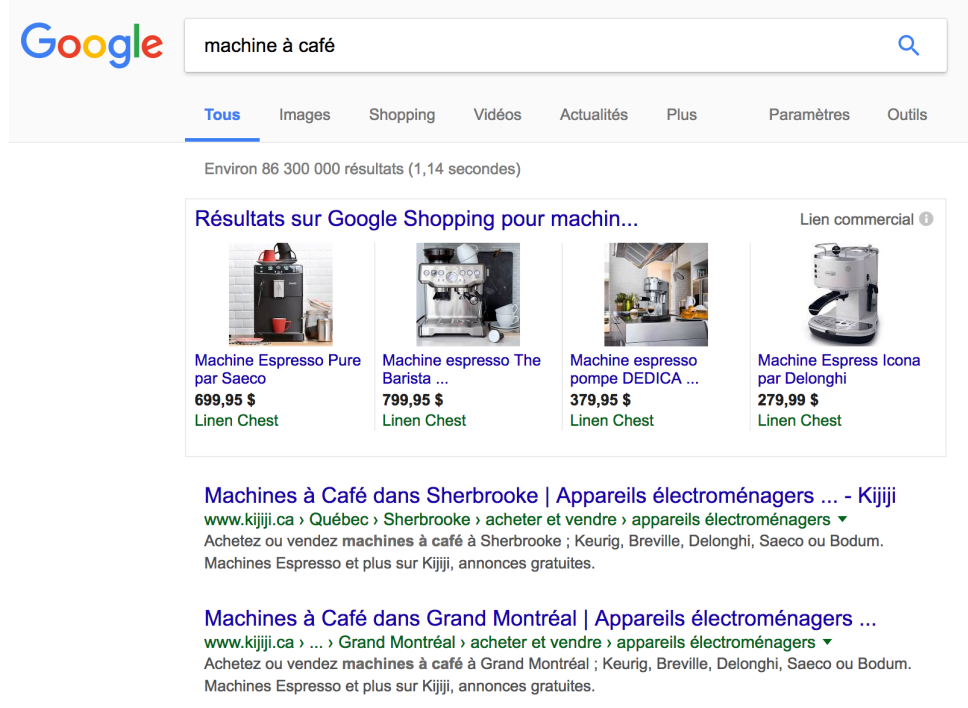

